\title{
三联噻吩-异噁唑、吡唑啉类化合物的合成与光活化性能研究
}

\author{
罗志刚刘正勇张广龙叶杰欣杨卓鸿* \\ (华南农业大学理学院应用化学系 广州 510642)
}

\begin{abstract}
摘要 $\alpha$-三联噻吩 $(\mathrm{a}-\mathrm{T})$ 是一类具有优异性能的光活化农药. 以三联噻吩为先导化合物, 经 $\mathrm{a}$ 醛基化制得关键中间体 2醛基三联噻吩, 然后与取代苯乙酮反应, 得到三联噻吩取代的 $\alpha, \beta$-不饱和酮, 再与盐酸差弪胺、水合肼关环, 最终合成两 类含 3,5-二芳基异噁唑和 3,5-二芳基吡唑啉的 $\alpha$-三联噻吩衍生物. 其结构经 ${ }^{1} \mathrm{H}$ NMR, IR 和元素分析确证. 初步生物活 性测定试验表明, 绝大多数目标化合物具有良好的光活化活性, 异趼㟇类衍生物的光活化活性普遍要好于吡唑啉类衍 生物, 其中化合物 $\mathbf{3 b}$ 光照前后的细胞毒杀活性差异为 64.06 倍. 但部分吡唑啉类衍生物整体显示出较高的细胞毒杀活 性，其中化合物 $\mathbf{4 d}$ 光照细胞毒杀活性为 $83.9 \%$.
\end{abstract}

关键词 $\alpha$-三联噻吩; 异噁唑; 吡唑啉; 斜纹夜蛾卵巢细胞; 光活化活性

\section{Synthesis and Phototoxic Activity of Isoxazole and Pyrazoline Derivatives Containing $\alpha$-Terthiophene}

\author{
Luo, Zhigang $\quad$ Liu, Zhengyong $\quad$ Zhang, Guanglong $\quad$ Ye, Jiexin $\quad$ Yang, Zhuohong* \\ (Department of Applied Chemistry, College of Science, South China Agricultural University, Guangzhou 510642)
}

\begin{abstract}
Terthiophene is a class of pesticides with predominant photoactivity. In this work, using terthiophene as the lead compound, the important mediator, 2-carbonyl terthiophene, was synthesized by the carbonylation. After reaction with substituted phenylethanone, terthiophene-substituted $\alpha, \beta$-unsaturated ketones were synthesized. Followed by the ring closure reaction with hydroxylamine hydrochloride and hydrazine, a series of 3,5-diaryl isoxazole and 3,5-diaryl pyrazoline drivatives containing $\alpha$-terthiophene were synthesized, respectively. Their structures were confirmed by ${ }^{1} \mathrm{H}$ NMR, IR and elemental analysis. The biological activity assay indicated that most of the synthesized compounds exhibit strong photoactivities. In addition, the photoactivities of isoxazole derivatives were much better than those of pyrazoline derivatives, where the photoactivity difference of compound $\mathbf{3 b}$ was 64.06 before and after illumination, especially. However, some of the pyrazoline derivatives showed higher activities of killing cells, where the activity of the compound $\mathbf{4 d}$ was as high as $83.9 \%$.
\end{abstract}

Keywords $\alpha$-terthiophene; isoxazole; pyrazoline; Spodoptera litura (SL) cell line; photoactivity

光活化农药是一类具有 “绿色农药”美称的新型、 高效、安全、低毒生物农药，它巧妙利用无所不在的自 然因子一光和氧成倍提高药效、发挥活性，同时促进自 身降解、避免残留, 从而使农作物生长的条件与农药发 挥药效的条件达到和谐统一. 与传统化学农药相比, 光 活化农药具有活性成分在自然界能彻底降解、广谱、高 效、抗性发展缓慢, 无交互抗性等诸多优点 ${ }^{[1 \sim 6]}$. 植物源 光活化农药 $\alpha$-三联噻吩 $(\alpha-\mathrm{T})$ 尤以高效、低毒、无残留而 成为其中的明星分子 ${ }^{[7 \sim 10]}$, 并在光电材料等方面展现出 潜在的应用前景 ${ }^{[11,12]}$. 国内外合成化学家以其为先导化
合物, 对其结构进行了活性修饰研究, 在保留 $\alpha$-三联噻 吩光活化性能的基础上，依据活性亚结构拼接方法和生 物等排原理, 引入具有良好生物活性的乙炔类官能团或 多元杂环如吡唑等 ${ }^{[11 ~ 20]}$, 以改进和提高三联噻吩类光 活化农药的光敏性和广谱活性.

本研究以 $\alpha$-三联噻吩为先导化合物, 制得关键中间 体 2-醛基三联噻吩, 然后与芳香酮反应, 得到 $\alpha, \beta$-不饱 和酮, 利用二元毒理拼接原理, 进一步与盐酸羟胺、水 合肼关环, 分别合成两类含 3,5-二芳基异噁唑和 3,5-二 芳基吡唑啉的 $\alpha$-三联噻吩衍生物. 同时选择斜纹夜蛾卵

\footnotetext{
*E-mail: yangzhuohong@aliyun.com; Tel.: 020-85280323; Fax: 020-85282366.

Received August 6, 2013; revised September 29, 2013; published online October 11, 2013.

Project supported by the National Natural Science Foundation of China (No. 21105030) and the Key Academic Program of 211 Project of South China Agricultural University (No. 2009B010100001).

国家自然科学基金(No. 21105030)和华南农业大学 211 三期建设(No. 2009B010100001)资助项目.
} 
巢细胞作为生物活性测试载体, 研究目标化合物的光活 化细胞毒杀活性, 探讨化合物结构对光活化活性的影 响. 合成路线见 Scheme 1.

\section{1 结果与讨论}

\section{1 波谱分析}

化合物 2a $\sim 2 \mathbf{e}$ 的 IR 光谱中 $3079.91 \sim 3064.48 \mathrm{~cm}^{-1}$ 为噻吩 $\alpha$ 位 $\mathrm{C}-\mathrm{H}$ 伸缩振动; $2923.7 \sim 2852.34 \mathrm{~cm}^{-1}$ 为噻 吩 $\beta$ 位 $-\mathrm{H}$ 伸缩振动; $1645 \sim 1650 \mathrm{~cm}^{-1}$ 为羰基的 $\mathrm{C}=\mathrm{O}$ 双 键的伸缩振动; 632.57 和 $536.14 \mathrm{~cm}^{-1}$ 为多联噻吩特有的 中间的噻吩环呼吸振动. ${ }^{1} \mathrm{H} N M R$ 谱中 $\mathrm{CH}=\mathrm{CH}$ 上 2 个 氢的化学位移分别在 $\delta 7.03 \sim 7.14$ 和 7.21 7.50 处, 由 于受苯环上不同取代基的影响, 化学位移有所改变.

化合物 3a 3e 的 IR 光谱中 $3174.06 \sim 3058.7 \mathrm{~cm}^{-1}$ 为噻吩 $\alpha$ 位 $\mathrm{C}-\mathrm{H}$ 伸缩振动; $2923.7 \mathrm{~cm}^{-1}$ 为异噁唑环上 $\mathrm{CH}=$ 的反对称伸缩, 1598.78 为异噁唑环 $\mathrm{C}=\mathrm{N}$ 的伸缩 振动, $1323.28 \sim 1336.49$ 与 $1274.78 \mathrm{~cm}^{-1}$ 为异噁唑环上 $\mathrm{CH}-\mathrm{O}$ 的伸缩振动吸收峰; $946.92 \mathrm{~cm}^{-1}$ 为异噁唑环上 $\mathrm{CH}=$ 的振动吸收峰; 698.14 和 $549.64 \mathrm{~cm}^{-1}$ 为多联噻吩 特有的中间的噻吩环呼吸振动. ${ }^{1} \mathrm{H}$ NMR 谱中, 异噁唑 环上 $\mathrm{CH}=$ 的化学位移大约在 $\delta 6.93 \sim 6.96$, 苯环上的不 同取代基对其化学位移也有一定影响.

化合物 $4 \mathrm{a} \sim 4 \mathrm{e}$ 的 IR 光谱中 $3434.77 \mathrm{~cm}^{-1}$ 为吡唑啉 环中缔合 $\mathrm{N}-\mathrm{H}$ 键的伸缩振动, 3103.06 和 $3058.7 \mathrm{~cm}^{-1}$ 为噻吩 $\alpha$-位 $\mathrm{C}-\mathrm{H}$ 伸缩振动; $2917.91 \mathrm{~cm}^{-1}$ 为吡唑啉环上 $\mathrm{CH}_{2}$ 的反对称伸缩; $2852.34 \mathrm{~cm}^{-1}$ 为吡唑啉环上 $\mathrm{CH}_{2}$ 的 对称伸缩; 1648.92 和 $1589.14 \mathrm{~cm}^{-1}$ 为吡唑啉环 $\mathrm{C}=\mathrm{N}$ 的 伸缩振动; $1452.21 \mathrm{~cm}^{-1}$ 为苯环骨架的伸缩振动; $1346.14,1319.14$ 与 $1236.21 \mathrm{~cm}^{-1}$ 为吡唑啉环上 $\mathrm{C}-\mathrm{N}$ 的 伸缩振动吸收峰; $829.28 \mathrm{~cm}^{-1}$ 为苯环对位取代的面外变 形振动吸收峰; 696.21 和 $540 \mathrm{~cm}^{-1}$ 为多联噻吩特有的中
间的噻吩环呼吸振动所作的贡献. ${ }^{1} \mathrm{H}$ NMR 谱中, 吡唑 啉环上 $\mathrm{CH}_{2}$ 化学位移分别在 $\delta 2.98 \sim 3.12$ 和 $3.50 \sim 3.55$ 左右, $\mathrm{CH}$ 的化学位移在 $\delta 5.14 \sim 5.20$ 左右, 受苯环上不 同取代基的影响, 化学位移有所改变.

\section{2 生物活性}

由华南农业大学天然药物与化学生物学教育部重 点实验室对目标化合物进行了光活化细胞毒杀活性测 定, 用 MTT 法测定 $24 \mathrm{~h}$ 对斜纹夜蛾卵巢细胞的抑制率. 药液浓度采用 $50 \mathrm{mg} / \mathrm{L}$, 测试方法见文献[21，22]，由飞 利浦紫外灯管提供 $365 \mathrm{~nm}$ 的紫外光光源, 测定结果见 表 1 , 根据下式计算细胞抑制率:

细胞活力 $\%=1$ - 调零后受试组 $\mathrm{OD}$ 值/调零后对照 组 OD 值

利用 DPS 软件 ${ }^{[23]}$ 统计分析不同处理的差异显著性 (Duncan's 新复极差测定法). 光活化细胞毒杀活性见表 1: 在黑暗条件下，除化合物 3e 的抑制率达到 $65.12 \%$ 外, 其余化合物抑制率均低于 $30 \%$. 但是，在光照条件下， 绝大多数化合物抑制活性明显增强，化合物 3a， 3c， 4d, $4 \mathrm{e}$ 的抑制率都超过 $70 \%$. 显示大部分目标化合物均具有 明显的光活化活性，其中 $3 \mathrm{~b}$ 光照前后的毒性相差 64 倍. 另值得注意的是化合物 $3 \mathrm{e}$ 光照前后活性均高于 $60 \%$. 但没有明显变化, 这意味着苯环上的取代基对光活化毒 性有着很重要的影响.

其次, 吡唑啉类衍生物整体显示出较为明显的细胞 毒杀活性, 其中化合物 4d 光照细胞毒杀活性为 $83.9 \%$. 化合物 3a $\sim 3 \mathrm{c}$ 的活性要高于 $\mathbf{4 a \sim 4 c}$, 而化合物 3d, 3e 则低于 4d, 4e. 黑暗条件下，化合物 $4 a \sim 4 d$ 高于 $3 a \sim$ 3d, 仅 3e 高于 4e. 数据说明异噁唑类化合物活性与吡 唑啉类化合物活性并无明显的对比关系，但异噁唑类化 合物光照条件下的细胞毒杀活性均衡性要好于吡唑啉

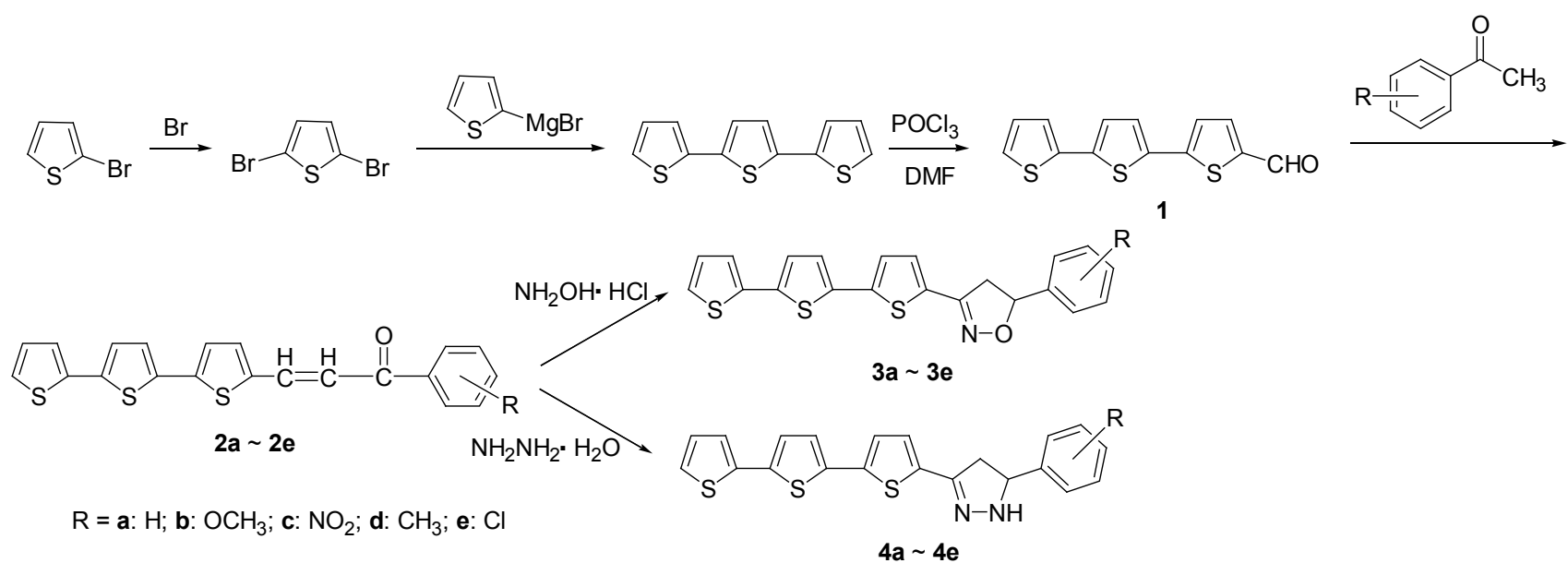

图 1 化合物 $2 \mathrm{a} \sim 2 \mathrm{e}, 3 \mathrm{a} \sim 3 \mathrm{e}$ 和 $4 \mathrm{a} \sim 4 \mathrm{e}$ 合成路线图

Figure 1 Synthetic route of $\mathbf{2 a} \sim 2 \mathrm{e}, 3 \mathrm{a} \sim 3 \mathrm{e}$ and $\mathbf{4 a} \sim \mathbf{4 e}$ 
类化合物.

表 1 化合物 $2 \mathrm{a} \sim 4 \mathrm{e}$ 对斜纹夜蛾卵巢细胞抑制测定结果 ${ }^{a}$

Table 1 Anti-spodoptera litura (SL) cell activity of compounds $\mathbf{2 a} \sim 4 \mathbf{e}$

\begin{tabular}{cccc}
\hline \multicolumn{4}{c}{ The inhibition rate $24 \mathrm{~h}(\%) \pm \mathrm{SE}$} \\
\hline \multirow{2}{*}{ Compound } & $\begin{array}{c}\text { Treatments with } \\
\text { Irradiation }\end{array}$ & $\begin{array}{c}\text { Treatments without } \\
\text { Irradiation }\end{array}$ & $\mathrm{I} / \mathrm{N}$ \\
\hline 2a & $59.51 \pm 4.34 \mathrm{a}$ & $1.56 \pm 1.56 \mathrm{~b}$ & 38.15 \\
$\mathbf{2 b}$ & $69.25 \pm 2.02 \mathrm{~b}$ & $2.40 \pm 1.83 \mathrm{c}$ & 28.85 \\
$\mathbf{2 c}$ & $53.83 \pm 1.56 \mathrm{~cd}$ & $3.38 \pm 0.55 \mathrm{c}$ & 15.93 \\
$\mathbf{2 d}$ & $42.95 \pm 3.14 \mathrm{e}$ & $0.84 \pm 0.93 \mathrm{c}$ & 51.13 \\
$\mathbf{2 e}$ & $59.51 \pm 4.33 \mathrm{c}$ & $1.56 \pm 1.30 \mathrm{c}$ & 38.15 \\
$\mathbf{3 a}$ & $71.09 \pm 1.43 \mathrm{a}$ & $1.87 \pm 0.75 \mathrm{~cd}$ & 38.02 \\
3b & $73.67 \pm 0.89 \mathrm{c}$ & $1.15 \pm 0.88 \mathrm{~d}$ & 64.06 \\
3c & $71.06 \pm 1.43 \mathrm{c}$ & $1.86 \pm 0.75 \mathrm{~cd}$ & 38.20 \\
$\mathbf{3 d}$ & $67.44 \pm 1.56 \mathrm{~d}$ & $4.71 \pm 1.13 \mathrm{c}$ & 14.32 \\
$\mathbf{3 e}$ & $63.97 \pm 1.64 \mathrm{e}$ & $65.12 \pm 1.56 \mathrm{~b}$ & 0.98 \\
4a & $63.43 \pm 2.27 \mathrm{e}$ & $8.19 \pm 2.20 \mathrm{de}$ & 7.74 \\
4b & $63.96 \pm 1.96 \mathrm{~d}$ & $3.64 \pm 0.51 \mathrm{bc}$ & 17.57 \\
4c & $55.61 \pm 2.75 \mathrm{e}$ & $8.10 \pm 0.95 \mathrm{bc}$ & 6.87 \\
4d & $83.90 \pm 2.31 \mathrm{ab}$ & $23.93 \pm 5.17 \mathrm{a}$ & 3.51 \\
4e & $78.91 \pm 2.09 \mathrm{~b}$ & $10.85 \pm 3.90 \mathrm{~b}$ & 7.27 \\
\hline
\end{tabular}

${ }^{a}$ Numbers followed by the same letter were data indistinctively different at $P=0.05$ level according to DMRT.

\section{2 结论}

本文以三联噻吩为先导化合物, 经不饱和酮中间 体, 与盐酸差弪胺、水合肼关环, 合成两类共 10 个三联噻 吩类衍生物. 测定了目标化合物对斜纹夜蛾卵巢细胞的 光活化毒杀活性, 结果显示大多数化合物都具有良好的 光活化活性, 异噁唑类衍生物的光活化活性普遍要好于 吡唑啉类衍生物, 但个别吡唑啉类衍生物显示出较高的 细胞毒杀活性, 其中化合物 $\mathbf{4 d}$ 光照细胞毒杀活性为 83.9\%. 显示苯环上的取代基对目标化合物的光活化毒 性差异有着重要影响.

\section{3 实验部分}

\section{1 仪器和试剂}

Yanaco CORDER MT-3 CHN 自动元素分析仪; Bruker AV-600 核磁共振仪, TMS 为内标; Nico let AVATAR 360 E.S.P. 型 Avatar FT-IR 红外光谱仪(KBr); X-4 数字显示显微熔点测定仪, 温度计未经校正; 实验 用的试剂和原料均为分析纯或化学纯, 使用前均经常规 处理. 中间体按文献[24 26]方法合成.

\section{2 中间体及目标化合物的合成}

\subsection{1 中间体 1 合成}

在 $50 \mathrm{~mL}$ 烧瓶内加入 $10 \mathrm{~mL} \mathrm{CH}_{2} \mathrm{Cl}_{2}$ 与 $1.2 \mathrm{~mL}(14.9$ $\mathrm{mmol}) \mathrm{N}, \mathrm{N}$ 二 二甲基甲酰胺后, 冰浴下搅拌, 混合均匀后, 向内逐滴加入 $1.2 \mathrm{~mL}(12.8 \mathrm{mmol}) \mathrm{POCl}_{3}$. 后将温度慢
慢加热到 $40{ }^{\circ} \mathrm{C}$, 得淡黄色溶液. 另在 $50 \mathrm{~mL}$ 三口烧瓶中 加入 $15 \mathrm{~mL} \mathrm{CH}_{2} \mathrm{Cl}_{2}, 3 \mathrm{~g}$ (12.1 mmol)的 $\alpha$-三联噻吩, 冰浴 下摚拌 $10 \mathrm{~min}$, 后向内逐滴滴加上述淡黄色溶液, 溶液 由无色变为黄色，滴加完毕，室温下反应 $2 \mathrm{~h}$.

后将反应液回流反应 $10 \mathrm{~h}$, 得红棕色溶液, 向内加 入 $10 \%$ 盐酸 $20 \mathrm{~mL}, 50{ }^{\circ} \mathrm{C}$ 条件下搅拌反应 $1.5 \mathrm{~h}$, 溶液分 层, 上层为水层, 下层为黑红色的有机相. 所得溶液冷 却至室温, $\mathrm{CH}_{2} \mathrm{Cl}_{2}$ 萃取. 脱溶剂, 烘干, 粗产物为 $2.71 \mathrm{~g}$. 将粗产物经柱层析分离, $\mathrm{CH}_{2} \mathrm{Cl}_{2}$ 进行洗脱, 得到 $1.76 \mathrm{~g}$ 亮黄色晶体，产率 $78.1 \%$. m.p. $135 \sim 136{ }^{\circ} \mathrm{C} ;{ }^{1} \mathrm{H}$ NMR $\left(\mathrm{CD}_{3} \mathrm{COCD}_{3}, 600 \mathrm{MHz}\right) \delta: 9.94$ (s, $\left.1 \mathrm{H}, \mathrm{CHO}\right), 7.94$ (d, $J=$ $3.6 \mathrm{~Hz}, 1 \mathrm{H}$, thiophene- $\mathrm{H}), 7.49 \sim 7.52(\mathrm{~m}, 3 \mathrm{H}$, thiophene-H), $7.39 \sim 7.40$ (dd, $J=3.6,3.8 \mathrm{~Hz}, 1 \mathrm{H}$, thiophene-H), 7.32 (d, $J=4.2 \mathrm{~Hz}, 1 \mathrm{H}$, thiophene-H), 7.12 7.14 (dd, $J=4.8,5.4 \mathrm{~Hz}, 1 \mathrm{H}$, thiophene-H); IR (KBr) v: 3432, 3093, 2923, 1651, 1458, 1443, 1223, 1049, 795 $\mathrm{cm}^{-1}$. Anal. calcd for C $56.49, \mathrm{H} 2.92$; found C $56.41, \mathrm{H}$ 2.87 .

\subsection{2 中间体 $\mathbf{2 a} \sim \mathbf{2} \mathrm{e}$ 合成}

$0.168 \mathrm{~g} \mathrm{KOH}\left(3 \mathrm{mmol}+2 \mathrm{~mL} \mathrm{H}_{2} \mathrm{O}\right)$ 溶液在室温下逐 滴加入 $2 \mathrm{mmol} 1$ 和 $2 \mathrm{mmol}$ 苯已酮的衍生物的 $25 \mathrm{~mL}$ 乙 醇溶液中, 反应混合液摚拌反应 $4 \mathrm{~h}$. 过夜后将反应液 倒入冰水, 并用盐酸中和, 然后过滤干燥, 柱层析分离, 即得产物.

1-苯基-3-(2'- $\alpha$-三联噻吩)-2-丙烯-1-酮(2a): 黄色粉 末, 产率 67\%. m.p. 162 164 ${ }^{\circ} \mathrm{C} ;{ }^{1} \mathrm{H}$ NMR $\left(\mathrm{CDCl}_{3}, 600\right.$ MHz) $\delta: 8.00 \sim 8.02(\mathrm{dd}, J=6.0,8.4 \mathrm{~Hz}, 2 \mathrm{H}, \mathrm{ArH}), 7.90$ (d, $J=15 \mathrm{~Hz}, 1 \mathrm{H}$, thiophene-H), 7.58 (t, $J=4.2 \mathrm{~Hz}, 1 \mathrm{H}$, $\operatorname{ArH}), 7.51$ (t, $J=4.2 \mathrm{~Hz}, 2 \mathrm{H}, \operatorname{ArH}), 7.30$ (d, $J=3.6 \mathrm{~Hz}$, $1 \mathrm{H}$, thiophene-H), $7.27(\mathrm{~d}, J=3.6 \mathrm{~Hz}, 1 \mathrm{H}$, thiophene-H), $7.25(\mathrm{~d}, J=3.6 \mathrm{~Hz}, 1 \mathrm{H}$, thiophene-H), 7.21 (dd, $J=3.2,3.6$ $\mathrm{Hz}, 1 \mathrm{H},=\mathrm{CH}), 7.18(\mathrm{~d}, J=3.6 \mathrm{~Hz}, 1 \mathrm{H}$, thiophene-H), $7.15(\mathrm{~d}, J=4.2 \mathrm{~Hz}, 1 \mathrm{H}$, thiophene-H), $7.12(\mathrm{~d}, J=3.6 \mathrm{~Hz}$, $1 \mathrm{H}$, thiophene-H), $7.04(\mathrm{q}, J=7.2 \mathrm{~Hz}, 1 \mathrm{H},=\mathrm{CH})$; IR (KBr) $v$ : 3042, 3072, 2793, 1649, 1585, 1456, 1336, 1221, 1047, 1014, 966, 835, 796, $692 \mathrm{~cm}^{-1}$. Anal. calcd for $\mathrm{C}_{21} \mathrm{H}_{14} \mathrm{OS}_{3}$ : C 66.63, H 3.73; found C 66.61, H 3.70.

1-(4-甲氧基苯基)-3-(2'- $\alpha$-三联噻吩)-2-丙烯-1-酤 (2b): 黄色固体，产率 $68.2 \%$. m.p. $155 \sim 158{ }^{\circ} \mathrm{C} ;{ }^{1} \mathrm{H}$ NMR $\left[\mathrm{CO}\left(\mathrm{CD}_{3}\right)_{2}, 600 \mathrm{MHz}\right] \delta: 8.13(\mathrm{~d}, J=7.2 \mathrm{~Hz}, 2 \mathrm{H}$, ArH), 7.88 (d, $J=15 \mathrm{~Hz}, 1 \mathrm{H}$, thiophene-H), 7.56 (t, $J=4.2$ $\mathrm{Hz}, 2 \mathrm{H}$, thiophene-H), $7.48(\mathrm{~d}, J=5.4 \mathrm{~Hz}, 1 \mathrm{H}$, thiophene-H), $7.38(\mathrm{~d}, J=1.2 \mathrm{~Hz}, 1 \mathrm{H}$, thiophene-H), 7.37 (d, $J=3.6 \mathrm{~Hz}, 1 \mathrm{H}$, thiophene- $\mathrm{H}), 7.36(\mathrm{dd}, J=3.2,3.6 \mathrm{~Hz}$, $1 \mathrm{H},=\mathrm{CH}), 7.29(\mathrm{~d}, J=3.6 \mathrm{~Hz}, 1 \mathrm{H}$, thiophene-H), $7.12(\mathrm{q}$, 
$J=7.2 \mathrm{~Hz}, 1 \mathrm{H},=\mathrm{CH}), 7.08(\mathrm{~d}, J=7.2 \mathrm{~Hz}, 2 \mathrm{H}, \operatorname{ArH})$, 3.92 (s, 3H, $\left.\mathrm{CH}_{3}\right)$; IR (KBr) v: 3429, 2923, 1645, 1589, 1398, 1336, 1218, 1093, 1010, 966, 829, 802, $694 \mathrm{~cm}^{-1}$. Anal. calcd for $\mathrm{C}_{22} \mathrm{H}_{16} \mathrm{O}_{2} \mathrm{~S}_{3}: \mathrm{C}$ 64.68, H 3.95; found $\mathrm{C}$ 64.65, H 3.97.

1-(4-硝基苯基)-3-(2'- $\alpha$-三联噻吩)-2-丙烯-1-酮(2c): 红色固体, 产率 $75.3 \%$. m.p. $180 \sim 182{ }^{\circ} \mathrm{C} ;{ }^{1} \mathrm{H}$ NMR $\left[\mathrm{CO}\left(\mathrm{CD}_{3}\right)_{2}, 600 \mathrm{MHz}\right] \delta: 8.41(\mathrm{~d}, J=9.0 \mathrm{~Hz}, 2 \mathrm{H}, \mathrm{ArH})$, $8.36(\mathrm{~d}, J=9.0 \mathrm{~Hz}, 2 \mathrm{H}, \mathrm{ArH}), 7.99(\mathrm{~d}, J=9.0 \mathrm{~Hz}, 1 \mathrm{H}$, thiophene-H), 7.63 (d, $J=3.6 \mathrm{~Hz}, 1 \mathrm{H}$, thiophene-H), 7.54 (d, $J=15 \mathrm{~Hz}, 1 \mathrm{H}$, thiophene-H), $7.50(\mathrm{q}, J=7.2 \mathrm{~Hz}, 1 \mathrm{H}$, $=\mathrm{CH}), 7.41(\mathrm{q}, J=3.6 \mathrm{~Hz}, 2 \mathrm{H}$, thiophene-H), 7.37 (d, $J=$ $3.6 \mathrm{~Hz}, 1 \mathrm{H}$, thiophene- $\mathrm{H}), 7.30(\mathrm{~d}, J=3.6 \mathrm{~Hz}, 1 \mathrm{H}$, thiophene-H), 7.13 (q, $J=7.2 \mathrm{~Hz}, 1 \mathrm{H},=\mathrm{CH})$; IR $(\mathrm{KBr}) v$ : 3429, 2923, 1645, 1589, 1398, 1336, 1219, 1093, 1010, 966, 829, 802, $694 \mathrm{~cm}^{-1}$. Anal. calcd for $\mathrm{C}_{21} \mathrm{H}_{13} \mathrm{NO}_{3} \mathrm{~S}_{3}: \mathrm{C}$ 59.55, H 3.09, N 3.31; found C 59.53, H 3.07, N 3.35.

1-(4-甲基苯基)-3-(2'- $\alpha$-三联噻吩)-2-丙烯-1-酮(2d): 黄色固体, 产率 $87.6 \%$. m.p. $143 \sim 145{ }^{\circ} \mathrm{C} ;{ }^{1} \mathrm{H}$ NMR $\left[\mathrm{CO}\left(\mathrm{CD}_{3}\right)_{2}, 600 \mathrm{MHz}\right] \delta: 8.03(\mathrm{~d}, J=8.4 \mathrm{~Hz}, 2 \mathrm{H}, \mathrm{Ar}-\mathrm{H})$, 7.89 (d, $J=15 \mathrm{~Hz}, 1 \mathrm{H}$, thiophene-H), 7.58 (t, $J=4.2 \mathrm{~Hz}$, $1 \mathrm{H}, \mathrm{ArH}), 7.54$ (t, $J=4.2 \mathrm{~Hz}, 2 \mathrm{H}, \mathrm{ArH}), 7.39$ (dd, $J=3.2$, $3.6 \mathrm{~Hz}, 1 \mathrm{H}$, thiophene-H), $7.27(\mathrm{~d}, J=7.18 \mathrm{~Hz}, 1 \mathrm{H}$, thiophene-H), 7.38 (d, $J=1.8 \mathrm{~Hz}, 1 \mathrm{H}$, thiophene-H), 7.36 (dd, $J=2.1,2.4 \mathrm{~Hz}, 1 \mathrm{H},=\mathrm{CH}), 7.36(\mathrm{~d}, J=3.6 \mathrm{~Hz}, 1 \mathrm{H}$, thiophene-H), 7.36 (d, $J=1.2 \mathrm{~Hz}, 1 \mathrm{H}$, thiophene-H), 7.29 (d, $J=3.6 \mathrm{~Hz}, 1 \mathrm{H}$, thiophene- $\mathrm{H}), 7.12(\mathrm{q}, J=7.2 \mathrm{~Hz}, 1 \mathrm{H}$, = $\mathrm{CH}), 2.80\left(\mathrm{~s}, 3 \mathrm{H}, \mathrm{CH}_{3}\right.$ ); IR (KBr) v: 3431, 2920, 1649, 1587, 1402, 1336, 1225, 1068, 1032, 1014, 966, 800, 688, $464 \mathrm{~cm}^{-1}$. Anal. calcd for $\mathrm{C}_{22} \mathrm{H}_{16} \mathrm{OS}_{3}$ : C 67.31, H 4.11; found $\mathrm{C} 67.33, \mathrm{H} 4.10$.

1-(4-氯苯基)-3-(2'- $\alpha$-三联噻吩)-2-丙烯-1-酮(2e): 黄 色固体, 产率 $81.5 \%$. m.p. $169 \sim 172{ }^{\circ} \mathrm{C} ;{ }^{1} \mathrm{H}$ NMR $\left[\mathrm{CO}\left(\mathrm{CD}_{3}\right)_{2}, 600 \mathrm{MHz}\right] \delta: 8.14(\mathrm{~d}, J=4.2 \mathrm{~Hz}, 2 \mathrm{H}, \mathrm{ArH})$, $7.93(\mathrm{~d}, J=15 \mathrm{~Hz}, 1 \mathrm{H}$, thiophene-H), $7.61(\mathrm{t}, J=4.2 \mathrm{~Hz}$, $2 \mathrm{H}, \mathrm{ArH}), 7.60$ (q, $J=7.2 \mathrm{~Hz}, 1 \mathrm{H},=\mathrm{CH}), 7.54(\mathrm{~d}, J=1.2$ $\mathrm{Hz}, 1 \mathrm{H}$, thiophene-H), $7.49(\mathrm{dd}, J=4.8,5.4 \mathrm{~Hz}, 1 \mathrm{H}$, thiophene-H), 7.40 (d, $J=1.2 \mathrm{~Hz}, 1 \mathrm{H}$, thiophene-H), 7.39 (d, $J=0.6 \mathrm{~Hz}, 1 \mathrm{H}$, thiophene-H), $7.36(\mathrm{dd}, J=3.1,3.6 \mathrm{~Hz}$, $1 \mathrm{H}$, thiophene- $\mathrm{H}), 7.30(\mathrm{~d}, J=3.6 \mathrm{~Hz}, 1 \mathrm{H}$, thiophene- $\mathrm{H})$, 7.13 (q, $J=7.2 \mathrm{~Hz}, 1 \mathrm{H},=\mathrm{CH})$; IR (KBr) v: 3429, 2923, 1645, 1589, 1398, 1336, 1219, 1093, 1031, 1010, 966, 829, $802,694 \mathrm{~cm}^{-1}$. Anal. calcd for $\mathrm{C}_{21} \mathrm{H}_{13} \mathrm{ClOS}_{3}: \mathrm{C} 61.08, \mathrm{H}$ 3.17; found C 61.07, H 3.19.

\subsection{3 目标化合物 $\mathbf{3 a} \sim \mathbf{3 e}$ 合成}

在 $50 \mathrm{~mL}$ 的圆底烧瓶中将上面得到的中间体查尔 酮 $2.0 \mathrm{mmol} \mathbf{2 a} \sim 2 \mathbf{e}$ 溶解于 $20 \mathrm{~mL}$ 吡啶中, 摚拌均匀后 加入 $2.0 \mathrm{mmol}$ 盐酸羟氨, 将反应液加热回流 $7 \mathrm{~h}$, 后冷 却倒入冰水中, 有固体沉淀生成, 过滤干燥, 柱层析分 离, 即得产物 $3 \mathbf{a} \sim 3 \mathbf{3 e}$.

3-苯基-5-(2'- $\alpha$-三联噻吩)-异噁唑(3a): 黄色固体, 产率 $83.4 \%$. m.p. $166 \sim 168{ }^{\circ} \mathrm{C} ;{ }^{1} \mathrm{H}$ NMR $\left[\mathrm{CO}\left(\mathrm{CD}_{3}\right)_{2}, 600\right.$ $\mathrm{MHz}] \delta: 7.50 \sim 7.51(\mathrm{~m}, 2 \mathrm{H}, \mathrm{ArH}), 7.48(\mathrm{~d}, J=1.2 \mathrm{~Hz}, 1 \mathrm{H}$, thiophene-H), $7.44 \sim 7.47$ (m, 3H, ArH), $7.33 \sim 7.35$ (dd, $J=3.2,3.6 \mathrm{~Hz}, 1 \mathrm{H}$, thiophene-H), $7.32(\mathrm{~d}, J=3.6 \mathrm{~Hz}, 1 \mathrm{H}$, thiophene-H), 7.26 (q, $J=16.2 \mathrm{~Hz}, 2 \mathrm{H}$, thiophene-H), 7.20 (d, $J=3.6 \mathrm{~Hz}, 1 \mathrm{H}$, thiophene-H), 7.10 (q, $J=8.0 \mathrm{~Hz}, 1 \mathrm{H}$, thiophene-H), 6.95 (q, $J=16.2 \mathrm{~Hz}, 1 \mathrm{H},=\mathrm{CH})$; IR $(\mathrm{KBr})$ $v: 3242,3174,3059,2924,1599,1423,1336,1255,1099$, 1041, 947, 916, 835, 795, $467 \mathrm{~cm}^{-1}$. Anal. calcd for $\mathrm{C}_{21} \mathrm{H}_{13} \mathrm{NOS}_{3}$ : C 64.42, H 3.35, N 3.58; found C 64.43, H $3.31, \mathrm{~N} 3.59$.

3-(4-甲氧基苯基)-5-(2'- $\alpha$-三联噻吩)-异噁唑(3b): 黄色固体, 产率 $78.6 \%$. m.p. $160 \sim 162{ }^{\circ} \mathrm{C} ;{ }^{1} \mathrm{H}$ NMR $\left[\mathrm{CO}\left(\mathrm{CD}_{3}\right)_{2}, 600 \mathrm{MHz}\right] \delta: 7.47 \sim 7.49(\mathrm{dd}, J=3.2,3.6 \mathrm{~Hz}$, $1 \mathrm{H}$, thiophene-H), $7.44(\mathrm{~d}, J=8.4 \mathrm{~Hz}, 2 \mathrm{H}, \mathrm{ArH}), 7.34 \sim$ 7.35 (dd, $J=3.2,3.6 \mathrm{~Hz}, 1 \mathrm{H}$, thiophene-H), 7.33 (d, $J=3.6$ $\mathrm{Hz}, 1 \mathrm{H}$, thiophene-H), $7.26 \sim 7.28(\mathrm{~m}, 2 \mathrm{H}$, thiophene- $\mathrm{H})$, 7.20 (d, $J=4.2 \mathrm{~Hz}, 1 \mathrm{H}$, thiophene-H), 7.12 (q, $J=8.0 \mathrm{~Hz}$, $1 \mathrm{H}$, thiophene-H), $7.01(\mathrm{~d}, J=9.0 \mathrm{~Hz}, 2 \mathrm{H}, \operatorname{ArH}), 6.96(\mathrm{q}$, $J=16.2 \mathrm{~Hz}, 1 \mathrm{H},=\mathrm{CH}), 3.86\left(\mathrm{~s}, 3 \mathrm{H}, \mathrm{OCH}_{3}\right)$; IR $(\mathrm{KBr}) v$ : 3421, 2924, 1605, 1512, 1439, 1329, 1232, 1174, 1095, 966, 922, 839, 795, 688, $588 \mathrm{~cm}^{-1}$. Anal. calcd for $\mathrm{C}_{22} \mathrm{H}_{15} \mathrm{NO}_{2} \mathrm{~S}_{3}$ : C 62.68, H 3.59, N 3.32; found C 62.67, H $3.56, \mathrm{~N} 3.31$.

3-(4-硝基苯基)-5-(2'- $\alpha$-三联噻吩)-异噁唑(3c)：红 色固体, 产率 75.3\%. m.p. $98 \sim 100{ }^{\circ} \mathrm{C} ;{ }^{1} \mathrm{H}$ NMR $\left[\mathrm{CO}\left(\mathrm{CD}_{3}\right)_{2}, 600 \mathrm{MHz}\right] \delta: 8.40(\mathrm{~d}, J=9.0 \mathrm{~Hz}, 2 \mathrm{H}, \mathrm{ArH})$, $8.06(\mathrm{~d}, J=9.0 \mathrm{~Hz}, 2 \mathrm{H}, \mathrm{ArH}), 8.04(\mathrm{~d}, J=9.0 \mathrm{~Hz}, 1 \mathrm{H}$, thiophene-H), 7.45 (d, $J=3.6 \mathrm{~Hz}, 1 \mathrm{H}$, thiophene-H), 7.41 (q, 2H, thiophene- $\mathrm{H}), 7.37(\mathrm{~d}, J=3.6 \mathrm{~Hz}, 1 \mathrm{H}$, thiopheneH), $7.30(\mathrm{~d}, J=3.6 \mathrm{~Hz}, 1 \mathrm{H}$, thiophene-H), 7.27 (d, $J=1.8$ $\mathrm{Hz}, 1 \mathrm{H}$, thiophene-H), $6.94 \sim 6.97(\mathrm{q}, J=16.2 \mathrm{~Hz}, 1 \mathrm{H},=$ $\mathrm{CH})$; IR (KBr) v: 3423, 3109, 2924, 1659, 1601, 1520, 1344, 1209, 1109, 1045, 959, 854, 795, $698 \mathrm{~cm}^{-1}$. Anal. calcd for $\mathrm{C}_{21} \mathrm{H}_{12} \mathrm{~N}_{2} \mathrm{O}_{3} \mathrm{~S}_{3}$ : C 57.78, H 2.77, N 6.42; found $\mathrm{C}$ 57.77, H 2.79, N 6.41.

3-(4-甲基苯基)-5-(2'- $\alpha$-三联噻吩)-异噁唑(3d)：黄 色固体, 产率 $82.3 \%$. m.p. $160 \sim 162{ }^{\circ} \mathrm{C} ;{ }^{1} \mathrm{H}$ NMR 
$\left[\mathrm{CO}\left(\mathrm{CD}_{3}\right)_{2}, 600 \mathrm{MHz}\right] \delta: 7.47 \sim 7.49(\mathrm{dd}, J=4.8,5.4 \mathrm{~Hz}$, $1 \mathrm{H}$, thiophene-H), 7.47 (d, $J=3.6 \mathrm{~Hz}, 1 \mathrm{H}$, thiophene-H), 7.39 (d, $J=7.8 \mathrm{~Hz}, 2 \mathrm{H}, \mathrm{ArH}), 7.34$ (dd, $J=3.2,3.6 \mathrm{~Hz}$, $1 \mathrm{H}$, thiophene-H), 7.33 (d, $J=4.2 \mathrm{~Hz}, 1 \mathrm{H}$, thiophene-H), 7.27 (d, $J=7.2 \mathrm{~Hz}, 2 \mathrm{H}, \operatorname{ArH}), 7.28$ (d, $J=1.8 \mathrm{~Hz}, 1 \mathrm{H}$, thiophene-H), 7.20 (d, $J=3.6 \mathrm{~Hz}, 1 \mathrm{H}$, thiophene-H), 7.12 (q, $J=8.0 \mathrm{~Hz}, 1 \mathrm{H}$, thiophene-H), 6.95 (q, $J=16.2 \mathrm{~Hz}, 1 \mathrm{H}$, $=\mathrm{CH}), 2.39\left(\mathrm{~s}, 3 \mathrm{H}, \mathrm{CH}_{3}\right)$; IR $(\mathrm{KBr})$ v: 3248, 2922, 2854, 1601, 1425, 1336, 1257, 1095, 1063, 955, 822, 793, 692, $467 \mathrm{~cm}^{-1}$. Anal. calcd for $\mathrm{C}_{22} \mathrm{H}_{15} \mathrm{NOS}_{3}$ : C 65.15, H 3.73, N 3.45; found C 65.13, H 3.71, N 3.47.

3-(4-氯苯基)-5-(2'- $\alpha$-三联噻吩)-异噁唑(3e): 黄色 固体，产率 89.8\%. m.p. $150 \sim 152{ }^{\circ} \mathrm{C} ;{ }^{1} \mathrm{H}$ NMR $\left[\mathrm{CO}\left(\mathrm{CD}_{3}\right)_{2}, 600 \mathrm{MHz}\right] \delta: 8.14(\mathrm{~d}, J=4.2 \mathrm{~Hz}, 2 \mathrm{H}, \mathrm{ArH})$, 7.94 (d, $J=15 \mathrm{~Hz}, 1 \mathrm{H}$, thiophene-H), 7.61 (t, $J=3.6 \mathrm{~Hz}$, 2H, ArH), 7.34 (dd, $J=3.2,3.6 \mathrm{~Hz}, 1 \mathrm{H}$, thiophene-H), $7.33(\mathrm{~d}, J=3.6 \mathrm{~Hz}, 1 \mathrm{H}$, thiophene-H), $7.26 \sim 7.28(\mathrm{~m}, 2 \mathrm{H}$, thiophene-H), 7.20 (d, $J=4.2 \mathrm{~Hz}, 1 \mathrm{H}$, thiophene-H), 7.12 (q, $J=8.0 \mathrm{~Hz}, 1 \mathrm{H}$, thiophene-H), 6.95 (q, $J=16.2 \mathrm{~Hz}, 1 \mathrm{H}$, $=\mathrm{CH})$; IR (KBr) v: 3384, 2976, 2924, 1597, 1491, 1425, 1398, 1331, 1095, 1047, 959, 835, 793, 692, $517 \mathrm{~cm}^{-1}$. Anal. calcd for $\mathrm{C}_{21} \mathrm{H}_{12} \mathrm{ClNOS}_{3}$ : C 59.21, H 2.84, N 3.29; found C 59.23, H 2.87, N 3.26.

\subsection{4 目标化合物 $\mathbf{4 a} \sim \mathbf{4 e}$ 合成}

在 $50 \mathrm{~mL}$ 的圆底烧瓶中将上面得到的中间体查尔 酮 $2.0 \mathrm{mmol} \mathbf{2} \mathbf{a} \sim \mathbf{2 e}$ 溶解于 $20 \mathrm{~mL}$ 吡啶中, 搅拌均匀后 加入 $2.0 \mathrm{mmol}$ 水和肼, 反应液加热回流 $2 \sim 3 \mathrm{~h}$, 冷却后 倒入冰水中, 有固体沉淀生成, 过滤干燥, 柱层析分离, 既得产物 $\mathbf{4 a} \sim \mathbf{4 e}$.

3-苯基-5-(2'- $\alpha$-三联噻吩)-吡唑啉(4a): 黄色固体, 产率 83.68\%. m.p. $156 \sim 158{ }^{\circ} \mathrm{C} ;{ }^{1} \mathrm{H}$ NMR $\left(\mathrm{CDCl}_{3}, 600\right.$ $\mathrm{MHz}) \delta: 7.71 \sim 7.73(\mathrm{~m}, 2 \mathrm{H}, \mathrm{ArH}), 7.43(\mathrm{dd}, J=4.3,4.8$ $\mathrm{Hz}, 1 \mathrm{H}$, thiophene-H), $7.39 \sim 7.42(\mathrm{~m}, 3 \mathrm{H}, \mathrm{ArH}), 7.29(\mathrm{dd}$, $J=3.2,3.6 \mathrm{~Hz}, 1 \mathrm{H}$, thiophene-H), 7.20 (d, $J=3.6 \mathrm{~Hz}, 1 \mathrm{H}$, thiophene-H), 7.18 (d, $J=4.2 \mathrm{~Hz}, 1 \mathrm{H}$, thiophene-H), 7.15 (d, $J=3.6 \mathrm{~Hz}, 1 \mathrm{H}$, thiophene-H), 7.09 (q, $J=8.0 \mathrm{~Hz}, 1 \mathrm{H}$, thiophene-H), 7.04 (d, $J=3.6 \mathrm{~Hz}, 1 \mathrm{H}$, thiophene-H), 5.20 (q, $J=6.0 \mathrm{~Hz}, 1 \mathrm{H}, \mathrm{CH}), 3.55$ (q, $J=7.2 \mathrm{~Hz}, 1 \mathrm{H}, \mathrm{CH}_{2}$ ), 3.05 (q, $J=4.2 \mathrm{~Hz}, 1 \mathrm{H}, \mathrm{CH}_{2}$ ); IR (KBr) v: 3435, 3280, 3103, 2917, 1649, 1589, 1446, 1346, 1236, 1186, 1055, 829, 796, 763, $696 \mathrm{~cm}^{-1}$. Anal. calcd for $\mathrm{C}_{21} \mathrm{H}_{16} \mathrm{~N}_{2} \mathrm{~S}_{3}$ : C 64.25, H 4.11, N 7.14; found C 64.20, H 4.13, N 7.11.

3-(4-甲氧基苯基)-5-(2'- $\alpha$-三联噻吩)-吡唑啉(4b): 黄色固体，产率 $74.3 \%$. m.p. $148 \sim 150{ }^{\circ} \mathrm{C} ;{ }^{1} \mathrm{H} \mathrm{NMR}$ $\left(\mathrm{CDCl}_{3}, 600 \mathrm{MHz}\right) \delta: 7.66(\mathrm{~d}, J=9.0 \mathrm{~Hz}, 2 \mathrm{H}, \mathrm{ArH}), 7.44$ (dd, $J=4.8,5.4 \mathrm{~Hz}, 1 \mathrm{H}$, thiophene-H), 7.30 (dd, $J=3.2$, $3.6 \mathrm{~Hz}, 1 \mathrm{H}$, thiophene-H), 7.21 (d, $J=3.6 \mathrm{~Hz}, 1 \mathrm{H}$, thiophene-H), 7.18 (d, $J=3.6 \mathrm{~Hz}, 1 \mathrm{H}$, thiophene-H), 7.16 (d, $J=3.6 \mathrm{~Hz}, 1 \mathrm{H}$, thiophene-H), $7.09 \sim 7.10$ (m, $1 \mathrm{H}$, thiophene-H), 7.04 (d, $J=3.6 \mathrm{~Hz}, 1 \mathrm{H}$, thiophene-H), 6.96 (d, $J=9.0 \mathrm{~Hz}, 2 \mathrm{H}, \mathrm{ArH}), 5.17$ (q, $J=6.0 \mathrm{~Hz}, 1 \mathrm{H}, \mathrm{CH}), 3.83$ (s, $3 \mathrm{H}, \mathrm{CH}_{3}$ ), 3.52 (q, $\left.J=7.2 \mathrm{~Hz}, 1 \mathrm{H}, \mathrm{CH}_{2}\right), 3.00$ (q, $J=4.2$ $\left.\mathrm{Hz}, 1 \mathrm{H}, \mathrm{CH}_{2}\right)$; IR (KBr) v: 3429, 3315, 3063, 2933, 1606, $1514,1419,1338,1248,1176,1051,820,692,534 \mathrm{~cm}^{-1}$. Anal. calcd for $\mathrm{C}_{22} \mathrm{H}_{18} \mathrm{~N}_{2} \mathrm{OS}_{3}$ : C 62.53, H 4.29, N 6.63; found $\mathrm{C} 62.57, \mathrm{H} 4.27, \mathrm{~N} 6.61$.

3-(4-硝基苯基)-5-(2'- $\alpha$-三联噻吩)-吡唑啉(4c): 红 色固体, 产率 87.6\%. m.p. 157 $159{ }^{\circ} \mathrm{C} ;{ }^{1} \mathrm{H} \mathrm{NMR}\left(\mathrm{CDCl}_{3}\right.$ $600 \mathrm{MHz}) \delta$ : 7.69 (d, $J=7.2 \mathrm{~Hz}, 2 \mathrm{H}, \mathrm{ArH}), 7.39$ (d, $J=7.8$ $\mathrm{Hz}, 2 \mathrm{H}, \mathrm{ArH}), 7.21$ (dd, $J=4.2,4.8 \mathrm{~Hz}, 1 \mathrm{H}$, thiophene-H), 7.15 (dd, $J=3.2,3.6 \mathrm{~Hz}, 1 \mathrm{H}$, thiophene- H), 7.05 (d, $J=$ $3.6 \mathrm{~Hz}, 1 \mathrm{H}$, thiophene-H), $7.02(\mathrm{~d}, J=3.6 \mathrm{~Hz}, 2 \mathrm{H}$, thiophene-H), 7.00 (d, $J=3.6 \mathrm{~Hz}, 1 \mathrm{H}$, thiophene-H), 6.91 (d, $J=3.6 \mathrm{~Hz}, 1 \mathrm{H}$, thiophene-H), $5.16(\mathrm{q}, 1 \mathrm{H}, \mathrm{CH}=), 3.46 \sim$ 3.50 (q, 1H, $\left.\mathrm{CH}_{2}\right), 3.15 \sim 3.19$ (q, $\left.1 \mathrm{H}, \mathrm{CH}_{2}\right)$; IR (KBr) v: 3387, 3226, 2928, 1595, 1514, 1423, 1338, 1173, 1109, 1062, 852, 791, 690, $467 \mathrm{~cm}^{-1}$. Anal. calcd for $\mathrm{C}_{21} \mathrm{H}_{15^{-}}$ $\mathrm{N}_{3} \mathrm{O}_{2} \mathrm{~S}_{3}$ : C 57.64, H 3.46, N 9.60; found C 57.61, H 3.47, $\mathrm{N} 9.61$.

3-(4-甲基苯基)-5-(2'- $\alpha$-三联噻吩)-吡唑啉(4d)：黄 色液体, 产率 $81.7 \%$. m.p. $136 \sim 138{ }^{\circ} \mathrm{C} ;{ }^{1} \mathrm{H} \mathrm{NMR}\left(\mathrm{CDCl}_{3}\right.$, $600 \mathrm{MHz}) \delta: 7.61$ (d, $J=7.8 \mathrm{~Hz}, 2 \mathrm{H}, \mathrm{ArH}), 7.44$ (dd, $J=$ 4.8, $5.4 \mathrm{~Hz}, 1 \mathrm{H}$, thiophene-H), 7.30 (dd, $J=3.6 \mathrm{~Hz}, 1 \mathrm{H}$, thiophene-H), 7.22 (d, $J=7.8 \mathrm{~Hz}, 2 \mathrm{H}, \mathrm{ArH}), 7.21$ (d, $J=$ $3.6 \mathrm{~Hz}, 1 \mathrm{H}$, thiophene-H), 7.18 (d, $J=3.6 \mathrm{~Hz}, 1 \mathrm{H}$, thiophene-H), 7.16 (d, $J=3.6 \mathrm{~Hz}, 1 \mathrm{H}$, thioph ene-H), 7.10 (q, $J=8.0 \mathrm{~Hz}, 1 \mathrm{H}$, thiophene-H), 7.04 (d, $J=3.6 \mathrm{~Hz}, 1 \mathrm{H}$, thiophene-H), 5.20 (m, 1H, CH), 3.53 (q, $J=7.2 \mathrm{~Hz}, 1 \mathrm{H}, \mathrm{CH}_{2}$ ), 3.02 (q, $\left.J=4.2 \mathrm{~Hz}, 1 \mathrm{H}, \mathrm{CH}_{2}\right), 2.35$ (s, 3H, $\left.\mathrm{CH}_{3}\right)$; IR (KBr) $v: 3429,3319,3081,2895,1736,1593,1510,1450,1342$, $1275,1057,819,793,480 \mathrm{~cm}^{-1}$. Anal. calcd for $\mathrm{C}_{22} \mathrm{H}_{18} \mathrm{~N}_{2} \mathrm{~S}_{3}$ : C 64.99, $\mathrm{H}$ 4.46, N 6.89; found $\mathrm{C} 64.97, \mathrm{H}$ 4.49, N 6.86 .

3-(4-氯苯基)-5-(2'- $\alpha$-三联噻吩)-吡唑啉(4e): 黄色 固体，产率 74.8\%. m.p. $150 \sim 152{ }^{\circ} \mathrm{C} ;{ }^{1} \mathrm{H} \mathrm{NMR}\left(\mathrm{CDCl}_{3}\right.$, $600 \mathrm{MHz}) \delta: 7.79$ (d, $J=8.4 \mathrm{~Hz}, 2 \mathrm{H}, \mathrm{ArH}), 7.42$ (d, $J=8.4$ $\mathrm{Hz}, 2 \mathrm{H}, \mathrm{ArH}), 7.36$ (dd, $J=4.2,4.8 \mathrm{~Hz}, 1 \mathrm{H}$, thiophene-H), 7.28 (dd, $J=3.2,3.6 \mathrm{~Hz}, 1 \mathrm{H}$, thiophene-H), 7.16 (d, $J=3.6$ $\mathrm{Hz}, 1 \mathrm{H}$, thiophene-H), $7.15(\mathrm{~d}, J=3.6 \mathrm{~Hz}, 2 \mathrm{H}$, thiophene$\mathrm{H}), 7.02 \sim 7.04(\mathrm{~m}, 2 \mathrm{H}$, thiophene-H), $5.27(\mathrm{~m}, 1 \mathrm{H}, \mathrm{CH}=)$, 
3.43 (q, $\left.J=7.2 \mathrm{~Hz}, 1 \mathrm{H}, \mathrm{CH}_{2}\right), 3.10(\mathrm{q}, J=4.2 \mathrm{~Hz}, 1 \mathrm{H}$, $\mathrm{CH}_{2}$ ); IR (KBr) v: 3440, 3313, 3064, 1591, 1495, 1425, 1402, 1352, 1273, 1093, 918, 827, 789, 692, $594 \mathrm{~cm}^{-1}$. Anal. calcd for $\mathrm{C}_{21} \mathrm{H}_{15} \mathrm{ClN}_{2} \mathrm{~S}_{3}$ : C 59.07, H 3.54, N 6.56; found C 59.05, H 3.57, N 6.54.

\section{References}

[1] Amor, B.; Jori, G. Insect Biochem. Mol. Biol. 2000, 30, 525.

[2] He, Y. P.; Ma, E. B.; Zhu, K. Y. Pestic. Biochem. Phys. 2004, 78, 103.

[3] John, G. V.; Graham, J. S.; Janet, H. Biochem. J. 2001, 357, 65.

[4] Luksiene, Z.; Kurilcik, N.; Jursenas, S.; Radziute, S.; Buda, V. J. Photochem. Photobiol. A 2007, 89, 15.

[5] Amini, J.; Sidovich, D. F. J. Plant Prot. Res. 2010, 50, 172.

[6] Achaleke, J.; Martin, T.; Ghogomu, R. T.; Vaissayre, M.; Brévault, T. Pest Manage. Sci. 2009, 65, 1147.

[7] Pernites, R.; Ponnapati, R.; Felipe, M. J.; Advincula, R. Biosens. Bioelectron. 2011, 26, 2766.

[8] Vladimír, C.; Jitka, V.; Lenka, K. Fitoterapia 2006, 77, 194.

[9] Collis, G. E.; Burrell, A. K.; Blandford, E. J.; Officer, D. L. Tetrahedron 2007, 63, 11141.

[10] Takahashi, H. T.; Britta, E. A.; Longhini, R.; Ueda-Nakamura, T.; De Mello, J. C. P.; Nakamura, C. V. Planta Med. 2013, 79, 330.

[11] Manca, P.; Scanu, R.; Zucca, A.; Sanna, G.; Spano, N.; Pilo, M. I. Polymer 2013, 54, 3504.

[12] Pardieu, E.; Saad, A.; Dallery, L.; Garnier, F.; Vedrine, C.; Hauquier, F.; Dalko, P.; Pernelle, C. Synth. Met. 2013, 171, 23.

[13] Ma, C. Q.; Mena-Osteritz, E.; Wunderlin, M.; Schulz, G.; Bauerle, P. Chem.-Eur. J. 2012, 18, 12880.

[14] Nivsarkar, M. Curr. Sci. 1999, 76, 1391.

[15] Yan, W. W.; Zhang, H.; Dong, Y. L.; Xu, H. H.; Zhang, Z. X. Chin. J. Pestic. Sci. 2012, 14, 277 (in Chinese).
(严汪汪, 张针, 董云龙, 徐汉虹, 张志祥, 农药学报, 2012, 14, 277.)

[16] Pu, W.; Zhang, Y. X.; Du, C.; Cheng, X. H. Chin. J. Pestic. Sci. 2012, 14, 341 (in Chinese).

(卜伟, 张宇轩, 杜超, 程晓红, 农药学报, 2012, 14, 341.)

[17] He, G. R.; Yang, J. M.; Wu, Y. F.; Yang, Z. H.; Liu, Z. Y.; Xu, H. H. Agrochemicals 2009, 48, 415 (in Chinese).

(何冠茹, 杨静美, 吴运福, 杨卓鸿, 刘正勇, 徐汉虹, 农药, 2009, 48, 415.)

[18] Jiang, Y.; Wang, J.; Zhong, Y. P.; Liu, P.; Deng, W. J. Acta Chim. Sinica 2012, 70, 103 (in Chinese).

(姜月, 王娟, 关丽, 钟一平, 刘平, 邓文基, 化学学报, 2012, 70, 103.)

[19] Singh, P.; Paul, K.; Holzer, W. J. Bioorg. Med. Chem. 2006, 14, 5016.

[20] Angemann, A.; Franke, H.; Preuss, R.; Hartfiel, U.; Wagner, H.; Auler, T.; Rosinger, C. US 6232271, 2001 [Chem. Abstr. 1999, 131, 5253].

[21] Huang, S. Q.; Xu, H. H.; Tong, S.; Zhang, Z. X. Acta Entamologica Sinica 2010, 53, 1104 (in Chinese). (黄素青, 徐汉虹, 童松, 张志祥, 昆虫学报, 2010, 53, 1104.)

[22] Wen, H. J.; Song, X. N.; Zhang, Z. X.; Cheng. D. M.; Zhang, Q. P. J. South China Agric. Univ. 2012, 33, 453 (in Chinese). (温好菊, 宋香宁, 张志祥, 程东美, 张清鹏, 华南农业大学学 报, 2012, 33, 453.)

[23] Wang, Y. J.; Zhang, Z. X.; Xu, H. H.; Liao, M. D.; Zhang, Q. F. Sci. Agric. Sinica 2010, 43, 1601 (in Chinese). (王玉健, 张志祥, 徐汉虹, 廖美德, 张前锋, 中国农业科学, 2010, 43, 1601.)

[24] Pham, C. V.; Burkhardt, A.; Shabanas, R. Phosphorus Sulfur 1989, $46,153$.

[25] Wei, Y.; Yang, Y.; Ye, J. M. Chem. Mater. 1996, 8, 2659.

[26] Rojas, J.; Dominguez, J. N.; Charris, J. E. Eur. J. Med. Chem. 2002, $37,699$.

(Li, L.; Lu, Z.) 